\title{
Apuntes para la concepción de Estado del proyecto político kirchnerista, a partir de Ley de Identidad de Género (2012)*
}

\author{
Andreina Colombo*
}

\section{Resumen}

Este artículo es un avance de investigación que tiene como objetivo examinar la concepción de Estado del proyecto político kirchnerista en la Ley de Identidad de Género (2012). Atendiendo a las tradiciones políticas a las que este apela en la relación Estado-ciudadanía, analizaremos el proceso desde el reclamo de la ciudadanía organizada hasta la aprobación de la ley.

Entendiendo la política como lucha de sentido, analizaremos tanto los acontecimientos como su legitimación. Para ello, aplicaremos una metodología cualitativa, valiéndonos del análisis de contenido de documentos y archivos oficiales, e información de medios de comunicación.

Así, identificamos elementos de la tradición liberal kantiana y otros de evocación nacional-popular. Sobre esta, identificamos metáforas articuladoras: reparación social, inclusión e igualdad. Estas suponen un Estado activo, sobre el que cobra identidad el conjunto del mapa de ideas. Precisamente, entendemos importante la resignificación del aseguramiento de la libertad individual con un Estado no-abstencionista.

\footnotetext{
* Artículo recibido el 4 de Mayo de 2015. Aceptado el 28 de Mayo de 2015.

** Andreina Colombo es Becaria de Beca Estímulo a la Vocación Científica (CIN) por la Facultad de Humanidades y Ciencias de la Universidad Nacional del Litoral.

Correo electrónico: colombo.andreina@gmail.com
} 


\section{Palabras clave}

Estado - ciudadanía - kirchnerismo.

\section{Abstract}

This paper is an advance research aims to examine the kirchnerism political proyect's State-concept; and here we will focus in the Law on Gender Identity (2012). In response to political traditions that appeals to the relatiochip Statecitizenship, we will analyze the process from organized citizens claim to the adoption of the law.

Understanding politics as a struggle for meaning, we will analyze events and their legitimacy. To do this, apply a qualitative methodology, availing ourselves of content analysis of official documents and files, and mass media information.

Thus, we identify elements of Kantian liberal tradition and other national-popular evocation. On this, we identified articulating metaphors: social repair, inclusion and equality. They assume an active state, on which the whole map of ideas make identity. Precisely understand the importance of redefinityng the assurance of individual freedom with a non-abstention State.

\section{Keywords}

State - citizenship - kirchnerism.

\section{Introducción}

Este artículo es un avance de investigación del trabajo de finalización de mi carrera de grado, por ello, aquí plasmaremos las líneas de análisis desarrolladas hasta el momento y las conclusiones preliminares de dicho proceso. ${ }^{1}$ Nuestro objetivo será el de identificar las tradiciones políticas a las que apela el proyecto político kirchnerista en su concepción de Estado, especialmente en relación con sectores de la ciudadanía, en el proceso de la Ley de Identidad de Género (LIG) aprobada en mayo de 2012.

Partiendo del planteo de Eduardo Rinesi (2011a; 2011b), entendemos que en la concepción de Estado del proyecto político kirchnerista conviven -no necesariamente de forma pacífica- elementos que provienen de diferentes corrientes del pensamiento político -que el autor denomina mix-, y el desafío de la Ciencia Política radica en dar cuenta de estas articulaciones. Asimismo, entendemos la política como creación radical,

\footnotetext{
${ }^{1}$ Este escrito es un avance de mi Trabajo Final de Carrera, para obtener la Licenciatura en Ciencia Política de la Universidad Nacional del Litoral. Asimismo, este proyecto de investigación es el que desarrollo en el marco de la Beca Estímulo a la Vocación Científica del Consejo Interuniversitario Nacional. En ambas instancias, cuento con la dirección de $\mathrm{Mg}$. Andrea Bolcatto, a la que estoy enormemente agradecida por el apoyo y la confianza constante en cada etapa de este desafío de investigación.
} 
en tanto "es una lucha por el sentido o lucha de valores contingente a través de la cual se da la constitución misma de la comunidad, los actores y las políticas" (Franzé, 2014: 13).

Por ello, pretendemos dar cuenta de la disputa en torno a la concepción de Estado del kirchnerismo, entendiendo que las metáforas políticas que le dan identidad se van resignificando y redelimitando a partir de sus relaciones con sectores de la ciudadanía. ${ }^{2}$ En este sentido, pensaremos la identidad política de un proyecto político atendiendo a las disputas de sentido que establecen con otros actores sociales, en condiciones históricamente situadas. Así, describiremos lo hecho en cuanto a la relación de la figura estatal con sectores de la ciudadanía en el proceso de la LIG, e indagaremos en las concepciones sobre el Estado presentes en lo dicho y lo escrito por integrantes del proyecto político kirchnerista.

A tal fin, aplicaremos una metodología cualitativa, valiéndonos de la técnica del análisis de contenido. De este modo, nos serviremos de fuentes secundarias, específicamente de documentos públicos y archivos oficiales, por un lado, e información de medios de comunicación, por otro. En cuanto a los primeros, utilizaremos proyectos legislativos, leyes, versiones taquigráficas de sesiones de ambas Cámaras, decretos y transcripciones de discursos oficiales del Poder Ejecutivo Nacional. Estos nos permitirán "conocer la perspectiva oficial del organismo o institución" (González Ríos, 1997: 245), así como de referentes del kirchnerismo. En cuanto al segundo tipo, manejaremos diarios de tirada nacional (Página 12, Clarín, La Nación) para "caracterizar un período de tiempo [...] establecer la trama general de los acontecimientos" (González Ríos, 1997: 249) y recoger declaraciones tanto de la ciudadanía como del proyecto político kirchnerista.

En cuanto a las categorías que guían el análisis, partimos de la definición de proyecto político de Amílcar Salas Oroño (2011), entendiéndolo como "determinadas 'metáforas políticas' que organizan la experiencia práctica a partir de un cierto mapa de ideas" (2011: 50). Asimismo, estas metáforas políticas definen la identidad de "un estilo de actuación en el poder [...y] la temporalidad política del propio proceso histórico de cada país" (Salas Oroño, 2011: 50). ${ }^{3}$ Así, concebimos al kirchnerismo bajo esta categoría con posterioridad al conflicto social originado a raíz de la Resolución 125 del Poder Ejecutivo Nacional en 2008, en tanto "es posible fijar ese momento como reordenador,

\footnotetext{
${ }^{2}$ Consideramos, evidentemente, que no solamente en la relación Estado-ciudadanía es que va tomando forma la metáfora política del kirchnerismo, pero el recorte que establecimos para esta investigación se centra en ella.

${ }^{3}$ Hacemos la salvedad de que en la definición de Salas Oroño parecería estar presente la idea de que el conjunto de conceptualizaciones que dan identidad a un proyecto político preceden a las decisiones políticas adoptadas, en tanto sería necesario preestablecerlas para poder organizar decisiones de acuerdo con ellas. Aquí, en cambio, entendemos que es necesario ser más flexibles en cuanto a este supuesto. Suponemos que la identidad política de un proyecto se va construyendo a partir de las luchas de sentido situadas históricamente, en una relación compleja entre metáforas políticas y acciones.
} 
para atrás y para adelante, respecto de lo que podría denominarse los contornos del proyecto político del kirchnerismo" (Salas Oroño, 2011: 52).

En cuanto al concepto de ciudadanía, empleamos la definición de Isidoro Cheresky (1998: 3): relación de los individuos con la vida política, que implica "un status que alude al conjunto de derechos garantizados por la ley" así como un sentimiento de interés y actividad efectiva en los asuntos públicos. Por ello, en nuestro análisis aplicaremos esta categoría al colectivo trans, ${ }^{4}$ en general, y a las organizaciones movilizadas, en particular.

\section{Concepciones de Estado en el proceso de la Ley de Identidad de Género}

\subsection{Comienzos del reclamo ciudadano por un derecho}

En primer término, repasaremos someramente los comienzos del reclamo por el derecho a la identidad de género desde determinados sectores ciudadanos. Nos referimos a la Federación Argentina de Lesbianas, Gays, Transexuales y Bisexuales (FALGTB) y el Frente Nacional por la Ley de Identidad de Género (FNLIG). Como consecuencia de su trabajo militante, desde 1999 hasta 2011 ingresaron ocho proyectos legislativos sobre la temática en la Cámara de Diputados. ${ }^{5}$ La FALGTB participó en la elaboración de la mayoría de estos, mientras que el FNLIG -que se formó a principios de 2010- lo hizo tan solo de uno, sobre el que se construyó el proyecto de consenso que finalmente se aprobó.

En estos procesos -ya sea dentro de la Cámara como fuera de ella-, rastrearemos elementos que nos permitan identificar concepciones de Estado, para luego ver de qué manera se inscriben dentro del mapa de ideas del kirchnerismo. En términos generales, distinguimos dos tradiciones políticas que nos permiten pensar el reclamo ciudadano por la LIG: una liberal-kantiana y otra posfundacional lefortiana.

En cuanto a la primera, refiere a los postulados políticos que derivan del pensamiento de Immanuel Kant, que parten de considerar que los hombres son portadores de razón, lo que les permite -casi que los obliga a- elegir el modo de vida que los haga más felices. Partiendo de esta autodeterminación individual, el reclamo por la LIG se piensa para "asegurar el reconocimiento de la dignidad, la singularidad y del propio proyecto de vida de las personas trans" (Proy. Ley de Identidad de Género, №5259-D-2007, Objetivos). En relación con esto, se realza la defensa de la libertad negativa ${ }^{6}$ y de la

\footnotetext{
${ }^{4}$ La palabra trans refiere al conjunto de personas que se autoidentifican como travestis, transexuales y transgénero.

${ }^{5}$ Los proyectos a los que hacemos referencia son: i) 0370-D-1999 (Alfredo Bravo-PS), ii) 5954-D-2003 (Ma. José Lubertino-PS), iii) 5259-D-2007 (Silvia Augsburger- PS), iv) 1736-D-2009 (reingresado por Augsburger), v) 7243-D-2010 (Silvana Guidici- UCR), vi) 7644-D-2010 (Juliana Di Tullio- FPV), vii) 8126-D2010 (Diana Conti-FPV) y viii) 5850-D-2011 (Marcelo López Arias-PJ de Salta).

${ }^{6}$ Sobre este concepto, tomamos la definición de Norberto Bobbio: "la situación en la cual un sujeto tiene la posibilidad de obrar o de no obrar, sin ser obligado a ello o sin que se lo impidan otros sujetos.... La
} 
esfera privada individual, en tanto "no existen razones jurídicas que permitan alguna clase de intromisión u obstrucción en el ejercicio del derecho a ser uno mismo sin causar un daño directo e inmediato a terceros" (Proy. Ley de Identidad de Género, N5259-D-2007, Fundamentos).

Asimismo, esta tradición permite pensar el reclamo ciudadano en relación con los derechos humanos", ya que "sin identidad no hay derechos civiles, y sin derechos civiles, no hay derechos humanos" (Claudia Pía Baudracco ${ }^{8}$, citada en el diario Página 12, 2009/11/07). En términos kantianos, estos son aquellos "válidos y exigibles universalmente" en tanto que "por fuente y por contenido, sólo pueden tener esa libertad radical que está relacionada con la esencia del ser racional como tal" (Hassner, 2009: 555). Por ello, concebimos que el carácter universal del derecho a la propia identidad de género se fundamenta en la libertad individual, y permite dejar de lado el enfoque de los derechos de las minorías para reclamar del cumplimiento del derecho básico de todo ser humano.

Desde estos argumentos en clave liberal-kantiana, las organizaciones usan el concepto de invisibilización civil de las personas trans, en tanto "sin una legislación que reconozca su identidad, son jurídicamente inexistentes" (Leclerc, 2014: 3). Aquí, ingresa el Estado con una carga negativa al instituirse como el ente que no permitía que cada persona determinase la identidad sexual que figura en su DNI.

En términos kantinos el Estado es Estado de Derecho, cuya función radica en asegurar leyes generales que permitan el ejercicio de la libertad individual. Bajo ninguna circunstancia una constitución política debe establecer normas que limiten los derechos fundamentales, siendo esta situación "el mayor despotismo pensable" (Kant, 2008: 37); en otros términos, ningún orden externo al individuo debe imponerle una particular visión acerca de la felicidad. La militante Marle Wayar, perteneciente al FNLIG, expresa este planteo: "El cambio de sexo tiene que ver con la potestad de lo académico que se yergue como despótica y que te dice que una mujer no puede ser sin vagina...Si alguien quiere transexualizarse está en su derecho" (citada en el diario Página 12, 2009/10/13). Así, desde esta tradición política se plantea como premisa que los derechos humanos son elementos legales que se anteponen frente o contra el Estado. De ello, el rol del Estado es pensado pasivamente, en tanto "la obligación fundamental

\footnotetext{
libertad negativa suele llamarse también «libertad como ausencia de impedimentos» 0 «libertad como ausencia de constricción»" (Bobbio, 1995: 97-98)

${ }^{7}$ Cuando usamos el concepto de derechos humanos, debemos ser conscientes de que su significación y alcances han variado a lo largo del tiempo y en relación con las luchas de sentido que se dieron en torno a él. Dicho en otros términos, hay que tener en cuenta que "los derechos humanos son 'históricos' en tanto [...] ha[n] sido fruto de un proceso 'gradual' motivado por luchas y defensas de 'nuevas' libertades ante los viejos poderes'" (Beriso y Quintana, 2010: 84)

${ }^{8}$ Claudia Pía Baudracco fue una militante y referente de la Asociación de Travestis, Transexuales y Transgénero (ATTTA), organización fundamental de este proceso dentro de la FALGTB.
} 
que había de cumplir para satisfacer aquellos derechos era la de omisión: no debía violarlos, ni impedir su goce, ni interferir en su ejercicio" (Bidart Campos, 2006: 211).

Al mismo tiempo que la tradición liberal-kantiana nos permite pensar predominantemente la concepción de Estado en la ciudadanía organizada, es necesario relativizar aquellos elementos que refieren al orden jurídico como finalidad exclusiva del Estado. Así, encontramos nociones en los discursos de los sectores ciudadanos que permiten ser pensados en clave posfundacional (Marchart, 2009), especialmente la conceptualización de democracia de Claude Lefort. Este autor "recupera el lugar de lo político [como] instituyente de una totalidad simbólica a partir de la producción de imaginarios" (Retamozo, 2009). Por ello, cuando el autor centra su análisis en la democracia otorga especial atención al orden simbólico que le es propio.

Así, la democracia es entendida como una forma de sociedad, es decir "más allá de un sistema de instituciones políticas históricamente determinadas" (Claude Lefort, citado en Gutiérrez, 2011: 248). Esta conceptualización nos habilita a pensar que el acceso a un nuevo DNI es tan solo el primer paso para el cumplimiento de los derechos humanos del colectivos trans. En este sentido, interpretamos las campañas mediáticas de visibilización de la problemática trans y de junta de firmas ${ }^{9}$ cuya finalidad no eran las instituciones estatales, sino impactar en las concepciones peyorativas en torno a las y los trans. Cuando una referente de esta comuniad argumenta que "[p]ara las personas trans, la democracia todavía no llegó" (Marcela Romero, citada en Leclerc, 2014: 6), está remitiendo a un Estado de Derecho todavía ausente al mismo tiempo que una sociedad aún discriminatoria.

Por ello, cobró importancia visibilizar la temática en la opinión pública, lo que podemos pensar bajo el concepto lefortiano de participación política, en tanto refiere al sentirse parte del juego político, es decir, el sentimiento de ser tenidos en cuenta en el debate sobre cuestiones políticas. Podemos pensar que los sectores ciudadanos se dieron estrategias de impacto social, de disputa del sentido, que los hicieron sentirse dentro de la discusión por esta ley -y veremos que luego participaron efectivamente en los procesos institucionales también. De esta manera, se remarca lo importante de "tener el sentimiento de formar parte y, más precisamente, el de tener derecho a tener derechos" (Lefort, 2011: 25).

\subsection{Procesamiento institucional e inscripción en la metáfora política kirchnerista}

Habiendo repasado las principales tradiciones políticas que nos permiten pensar el reclamo de la ciudadanía organizada por la LIG, pasaremos ahora al análisis de la

\footnotetext{
${ }^{9}$ En 2010, la FALGTB lanza una campaña de recolección de firmas en apoyo al proyecto ingresado en la Cámara de Diputados ese año (1736-D-2010) que se extiende a varios puntos del país. Un dato importante es que Aníbal Fernández "fue la primera persona que firmó la planilla de la campaña de juntas de firmas que se realizó para exigir el tratamiento de la Ley de Identidad de Género en el Congreso Nacional" (Leclerc, 2014: 10).
} 
manera en que estos sectores fueron relacionándose con el proyecto político kirchnerista. En este sentido, creemos necesario marcar un antecedente relevante: la Ley de Matrimonio Igualitario (LMI), de 2010. Con esto hacemos referencia a que la LMI permitió el acercamiento político perdurable entre las organizaciones de diversidad sexual (la FALGTB y la Comunidad Homosexual Argentina, que integrará luego el FNLIG) y el proyecto político kirchnerista.

De igual manera, en el proceso de debate social y parlamentario de la LMI se entabló un importante vínculo entre estas organizaciones y el Instituto Nacional contra la Discriminación, la Xenofobia y el Racismo (INADI). ${ }^{10}$ En este sentido, el INADI dio acompañamiento -más bien simbólico- en la estrategia judicial de las organizaciones de solicitar, mediante acciones de amparo, los cambios registrales de personas trans. Desde las áreas jurídicas de la FALGTB y de la CHA (como integrante del FNLIG), se gestionaron acciones de amparos desde 2010 hasta la aprobación de la LIG en 2012, teniendo como fundamentación el postulado liberal-kantiano de la autodeterminación personal.

Consideramos más bien simbólico el apoyo del kirchnerismo sobre esta cuestión, ya que las acciones judiciales fueron pensadas y propiciadas por las organizaciones de la sociedad civil, e integrantes del kirchnerismo estuvieron presentes en los actos de entrega de nuevos DNI obtenidos mediante sentencias. Los casos más emblemáticos fueron los de Tania Luna ${ }^{11}$-gestionado desde la CHA- y Florencia de la V (nombre artístico), gestionado desde FALGTB. En la entrega de ambos DNI se hicieron presentes referentes del INADI, así como funcionarios de gobierno (Aníbal Fernández, jefe de Gabinete, y Florencio Randazzo, ministro del Interior). Como analiza Leclerc (2010) refiriéndose al segundo caso, "la entrega de este documento por parte de estos altos funcionarios del Poder Ejecutivo en un acto que se celebró en la propia Casa de Gobierno, reflejó un firme compromiso y voluntad política con el reconocimiento de los derechos de la población trans" (2010: 10).

Estos actos de presencia de referentes del kirchnerismo, así como de un organismo que depende directamente del Poder Ejecutivo Nacional, podemos entenderlos como fotos del afianzamiento de una relación política construida en torno a la LIG. También podemos pensarlas como imágenes de la decisión política -o el riesgo, en términos de Corrales y Pecheny, 2010- de este proyecto político de aprobar este tipo de leyes de ampliación de derechos en el periodo de tiempo analizado.

\footnotetext{
${ }^{10}$ Este órgano estatal descentralizado se encuentra bajo la órbita del Ministerio de Justicia y Derechos Humanos de la Nación, por lo que son las autoridades del Poder Ejecutivo quienes designan a su director. Por ello es que consideramos al INADI como un actor dentro del proyecto político kirchnerista, sumamente relevante para el caso de análisis.

${ }^{11}$ El caso de esta actriz fue el primero de América Latina en que se consiguió el reconocimiento de su identidad de género sin someterse a una operación de reasignación sexual.
} 
En cuanto a la discusión parlamentaria propiamente dicha, comienza en agosto de 2011 en la Cámara Baja, trabajando conjuntamente con tres proyectos legislativos. ${ }^{12} \mathrm{EI}$ primero de ellos fue ingresado por diputados de la Unión Cívica Radical (UCR) sin el aval explícito de las organizaciones de diversidad sexual, aunque mantenía los postulados liberal-kantianos que analizamos. El segundo fue propuesto desde la FALGTB con el auspicio de la diputada Juliana Di Tullio del Frente para la Victoria (FPV) y las firmas de representantes de todos los bloques, excepto de la UCR. Finalmente, el último proyecto es propiciado por el FNLIG y auspiciado, también, por el FPV (diputada Diana Conti), y con la firma de los mismos bloques que el anterior. Esto último refuerza la idea del apoyo del oficialismo a este reclamo de las organizaciones desde mediados de 2010 que no se circunscribe exclusivamente a la FALGTB, al mismo tiempo que evidencia el grado de consenso que la LIG tenía entre todas las fuerzas políticas presentes en la Cámara de Diputados. ${ }^{13}$

Se genera un proyecto consensuado entre las comisiones de la Cámara Baja, el que se debate en la última sesión del período ordinario de 2011, obteniendo media sanción (168 votos a favor, 17 en contra y 7 abstenciones). Ya en el siguiente periodo legislativo, es debatido en la Cámara Alta el 9 de mayo, día en que es aprobado con 55 votos afirmativos y 1 abstención.

Tanto referentes de la FALGBT como del FNLIG fueron convocados a las discusiones de las comisiones que analizaron los proyectos, tanto en Diputados como en Senadores. Asimismo, en estas instancias estuvo presente el director del INADI, marcando nuevamente la importancia de esta institución en este proceso. Asimismo, en las palabras del funcionario podemos identificar elementos liberales (la visibilización civil como primer paso hacia los derechos humanos del colectivos trans) ${ }^{14}$, al mismo tiempo que comienzan a tomar relevancia algunos diferentes: "Nosotros somos los responsables y los actores principales para lograr la plena inclusión de todos y todas los que habitamos nuestro país" (Pedro Mouratian, citado en SentidoG, 2010/18/08).

Sobre esto último, es interesante la presencia de expresiones como "garantizar el cumplimiento" y "somos los responsables" las que explicitan la posición del locutor (mediando la primera persona del plural, nos) como parte del proyecto político gobernante. Se trata, además, de un Estado que se autopercibe con el compromiso de garantizar el cumplimiento de derechos. Asimismo, introduce una expresión recurrente

\footnotetext{
12 Hacemos referencia a: 7243-D-2010, 7644-D-2010 y 8126-D-2010, respectivamente.

${ }^{13}$ Este consenso se evidenció, asimismo, con la presencia de congresales de varias fuerzas políticas en el acto de la campaña de la FALGBT para instalar en la opinión pública la relevancia del derecho a la identidad de género (Diario Página 12, 2011/08/07), que tuvo lugar en el Congreso de la Nación.

14 "Hoy, una ley de Identidad de Género no representa solamente tener un DNI con el nombre que uno eligió, sino que debe ser el inicio de un camino que el Estado Nacional y la sociedad tienen que transitar para garantizar el cumplimiento de una serie de derechos económicos, sociales y culturales que le den dignidad plena a un colectivo que fue históricamente desplazado y vapuleado" (Pedro Mouratian, citado en SentidoG, 2010/18/08).
} 
en los discursos de los referentes kirchneristas: "lograr la plena inclusión". Así entendida, la inclusión es vista como uno de los compromisos del proyecto político gobernante con la sociedad civil. En este sentido, la inclusión puede pensarse como una de las ficciones orientadoras del proceso histórico que denominamos kirchnerismo.

Este planteo lo retomamos de Retamozo y Muñoz (2012), quienes argumentan que si bien no es posible pensar la política de la fuerza gobernante desde 2003 sin la "contingencia" -y acomodamiento- ante las situaciones imprevistas, es posible identificar cierta unidad significativa durante el conjunto del proceso, a partir de "ciertos significantes que actúan como ficciones orientadoras: inclusión, justicia social, democracia, igualdad, por ejemplo, que dotan de sentido ex post a las decisiones tomadas" (Muñoz \& Retamozo, 2012, pág. 3). Esta ficción orientadora la entendemos aquí como una de las metáforas del proyecto político kirchnerista, la que dentro de este mapa de ideas no es posible pensarla escindida del Estado, como detallaremos más adelante.

Tanto la letra de los dictámenes de las comisiones de ambas Cámaras como el acto mismo en que se aprobó la legislación, pusieron de manifiesto el amplio consenso que la LIG tenía entre todos los bloques de Congreso, el que excedía claramente al proyecto político kirchnerista. ${ }^{15}$ Asimismo, la participación activa de las organizaciones en el trabajo de las comisiones podemos entenderla como una manera en que el Estado se relaciona con sectores de la ciudadanía organizada, aunque no se podría atribuir exclusivamente a una fuerza política, como vimos. Sin embargo, fue la fuerza política gobernante la que logró inscribir más exitosamente esta ampliación de derechos dentro de las metáforas políticas que la identifican. Esta inscripción dentro del proyecto político kirchnerista la podremos evidenciar más claramente en las intervenciones en el debate parlamentario de la LIG, donde los representantes de esta fuerza política ponen en juego el reconocimiento a la militancia por los derechos trans, al mismo tiempo que defienden una concepción de Estado diferente a la predominante en estos sectores organizados. A continuación analizaremos las intervenciones de diputados y senadores del proyecto político kirchnerista, ${ }^{16}$ en las respectivas sesiones en que se trató la LIG en el Congreso. Teniendo en cuenta las tradiciones políticas a las que se apela discursivamente, observamos que persisten elementos liberales en las intervenciones parlamentarias. En primer lugar, se comparte la postura negativa sobre la manera en que el Estado ha actuado sobre esta cuestión, invisibilizando civilmente a las personas trans, al negarle el reconocimiento de su identidad: "cuando el Estado no los reconoce y

\footnotetext{
${ }^{15}$ Aquí es importante remarcar que la LIG contó con mayor consenso que la LMI. En la Cámara de Diputados el Matrimonio Igualitario tuvo 126 votos a favor, 110 en contra y 6 abstenciones; y en la Cámara de Senadores, 33 votos a favor, 27 en contra y 3 abstenciones.

${ }^{16}$ Del debate en la Cámara Baja, seleccionamos las intervenciones de los diputados Vilma Ibarra, Diana Conti, Juliana Di Tullio, Agustín Rossi, María Cristina Regazzoli y María Stella Córdoba. De las intervenciones en la Cámara Alta tomamos las de los senadores Aníbal Fernández, Inés Blas, Beatriz Rojkes de Alperobich, Ada Itúrrez de Capellini, Sonia Escudero, Ruperto Godoy, Elena Corregido.
} 
no los incluye en las leyes, termina avalando simbólicamente una discriminación que se convierte en violencia social en las calles" (Dip. V. Ibarra, en Cámara de Diputados, 30/11/2011).

Asimismo, se evidencia que el derecho a la propia identidad debe ser enmarcado en los derechos humanos. Igualmente, se establece aquel derecho civil como la llave maestra que permitirá el acceso a los demás derechos inalienables de los seres humanos: "para que el derecho a la igualdad y el ejercicio de los derechos humanos realmente existan, es necesario el reconocimiento a la identidad de género" (Sen. A. Itúrrez de Capellini, en Cámara de Senadores, 09/05/2012).

Dentro del proyecto político kirchnerista se enmarcó a la LIG en lo que denominan "política de derechos humanos" -anulación de las leyes de Obediencia Debida y Punto Final, juicio a los responsables de crímenes de lesa humanidad de la última dictadura militar, utilización de edificios donde funcionaban centros clandestinos de detención como espacios de memoria, entre otros-: "el derecho a la identidad de género forma parte, sin lugar a dudas, de la política de Derechos Humanos instaurada por el doctor Néstor Kirchner y profundizada por nuestra presidenta, Cristina Fernández de Kirchner" (Sen. I. Blas, en Cámara de Senadores, 09/05/2012). De esta manera, el argumento que había permitido a los sectores organizados de la ciudadanía incrementar su legitimidad en la sociedad civil y en los diálogos con representantes del Estado, permitió al kirchnerismo enmarcar a la LIG en otro conjunto de luchas políticas más amplias. En este sentido, el argumento liberal de los derechos humanos fue un importante elemento común, que permitió a ambos entablar relaciones de sentido legitimantes.

Asimismo, en las intervenciones analizadas se remarca la importancia de la no intromisión del Estado en la autodeterminación de cada ciudadano y el respeto irrestricto del ámbito de la libertad individual, siempre que no afecte los derechos de terceros: "pretendemos que si no hay afectación de derechos de terceros ni, en suma, otros derechos en juego más que la propia identidad y dignidad de la persona, el Estado reconozca y respete esa identidad" (Dip. V. Ibarra, en Cámara de Diputados, 30/11/2011). Así, se mantiene el planteo de que la LIG amplía los márgenes de la libertad individual, por lo que tomando esta decisión el Estado acciona como Estado de Derecho o Constitucional: "No queremos imponer un plan de vida a nadie... [eso sería] un Estado autoritario. En un Estado constitucional de derecho lo que valoramos es la diferencia" (Dip. V. Ibarra, en Cámara de Diputados, 30/11/2011).

Con todo, comienzan a evidenciarse argumentos que difieren respecto a la concepción liberal, especialmente en cuanto al rol que le corresponde a este Estado de Derecho. En las intervenciones, podemos observar que este ya no es considerado exclusivamente como garante del ejercicio de la libertad individual, sino que ellos se consideran partícipes de un Estado activo: "...[la LIG] no significa solamente el reconocimiento del derecho [...] se trata también de integrar [... que] desde el Estado le garanticemos a esas personas el acceso al goce de todos los derechos (Sen. E. 
Corregido, en Cámara de Senadores, 09/05/2012). El Estado es aquí comprendido como un actor que acciona sobre una realidad que se pretende modificar; es decir, un Estado que "se hace cargo"17 de que está "en deuda" con un sector de la ciudadanía excluido del acceso a un derecho básico, y por ello toma la decisión política de dictar una ley que resguarde la libertad individual, al mismo tiempo que se asegure el pleno ejercicio de estas prerrogativas. Este elemento dentro del conjunto del proyecto político kirchnerista lo podemos pensar como una de las recuperaciones de este proceso histórico (Rinesi; 2011b): el Estado. Más específicamente, Eduardo Rinesi refiere al retorno a la idea de que los derechos no se postulan frente o contra el actor estatal como vimos en la matriz liberal-, "sino -al revés- garantizados, por una activa presencia y un conjunto de acciones efectivas del Estado" (Rinesi, 2011b: 147).

También aquí podemos dar cuenta de la importancia que se atribuye al rol integrador, entendido como la tarea cuyo objetivo es que sectores excluidos del ejercicio de un derecho formen parte de la ciudadanía. En la LIG, la metáfora política de la inclusión tiene límites claramente definidos: las personas trans, antes apartadas del acceso de los derechos humanos más básicos, ahora podrán ser incluidos a partir del nuevo documento de identidad.

En este sentido, podemos pensar que el proyecto político ha logrado configurar una promesa, como exponen Muñoz y Retamozo (2012), la que "centró sus coordenadas en la re-significación del Estado como garante de la inclusión y reparador de los daños sociales en el marco de una evocación nacional-popular" (Muñoz \& Retamozo, 2012, pág. 2). Nos parece interesante poder enmarcar los elementos que venimos analizando dentro de una tradición nacional-popular, la que entenderemos como una matriz política que "tiende a sostenerse sobre el triple eje de la afirmación de la nación, el Estado redistributivo y conciliador, y el liderazgo carismático junto con las masas organizadas -el pueblo-" (Svampa, 2011: 18-19).

En esta clave es que podemos interpretar el planteo de un Estado activo presente en las intervenciones de legisladores kirchneristas que marcamos arriba. En este caso no hacemos referencia al Estado redistribuidor de ingresos, sino más bien en la garantía y ampliación de los derechos. Sobre esta cuestión Rabornikof y Aibar (2012) resaltan que esta nueva interpelación nacional-popular de ciertos gobiernos latinoamericanos del Siglo XXI viene de la mano de la ampliación de derechos ciudadanos, en temas "no tradicionales" como de diversidad sexual (pág. 66). Justamente, en esta línea de ampliación de ciudadanía es que podemos pensar el rol del Estado en el caso de la LIG.

Asimismo, continuando en línea con el planteo de la revalorización del papel estatal, Rabotnikof y Aibar (2012) entienden como fundamental que se retome la premisa del

\footnotetext{
${ }^{17}$ Parafraseando las palabras de Diana Conti en esta discusión: "Me gusta ver un Estado que se hace cargo de la riqueza en la diversidad. Entiendo que estamos dando siempre pasos, y este es un caso, para saldar deudas pendientes con un sector de la población que no goza del derecho de vivir tal como se autopercibe, tal cual se siente" (Honorable Cámara de Diputados, 30/11/2011)
} 
"Estado como un instrumento de la acción colectiva" (pág. 65). En este sentido, podemos interpretar el reconocimiento de la existencia de una demanda organizada desde la sociedad civil por esta ampliación de derechos, y de una tarea militante que no proviene de un partido político en particular. Así, la Dip. Vilma Ibarra expresa: "Estos dictámenes son el fruto de una lucha histórica de muchos años y han costado mucho sufrimiento. Por lo tanto, quiero brindar un reconocimiento a esa militancia" (Cámara de Diputados, 30/11/2011). Aquí es importante la referencia al origen de esta ley, en tanto la manera en que se inscribe dentro de la metáfora política kirchnerista tiene como un importante elemento que esta provenga del reclamo de sectores discriminados socialmente durante muchos años. Esto permite a la fuerza política gobernante sumar la legitimidad que las organizaciones han construido en el último tiempo, y la legitimidad propia de una ampliación de derechos. En este sentido, el Estado es puesto al servicio de las demandas ciudadanas, como el instrumento capaz de materializarlas. Asimismo, no se está negando o borrando la militancia de estos sectores no partidarios, sino más bien que se la está integrando dentro de un conjunto mayor de luchas de los excluidos; de esta manera es posible relacionarla, asimismo, con la metáfora de inclusión.

Continuando con el mapa de ideas sobre el rol activo del Estado, encontramos presente en cada una de las intervenciones parlamentarias analizadas, la evocación de otra metáfora: la igualdad. Si entendemos al Estado como un actor reparador de daños, podemos pensar la inclusión como la manera a partir de la cual subsanar las exclusiones sociales, y la igualdad como un valor que guía las medidas tomadas al respecto: "seguimos construyendo una sociedad mejor, más inclusiva, más tolerante y menos discriminatoria" (Dip. A. Rossi, en Cámara de Diputados, 30/11/2011).

Continuando con el análisis, esta evocación nacional-popular de la que veníamos hablando se mantiene en el discurso que diera Cristina Fernández ese día. En primer término es importante notar que esta matriz está mechada con argumentos que pueden ser pensados en clave liberal. Sin embargo, predominan las metáforas políticas de reparación social e igualdad: "esta es la sociedad que queremos, una sociedad de reparación por todo lo que tuvieron que pasar hasta llegar a este momento de igualdad" (Fernández C. , 2012/07/02).

Así, podemos identificar tres importantes elementos de la evocación nacional-popular del proyecto político kirchnerista: reparación social, inclusión e igualdad. Estas tres ficciones orientadoras tienen como denominador común el supuesto de un Estado activo, eje a partir del cual logran articularse el resto de los elementos del mapa de ideas.

\section{A modo de recapitulación}

Hasta aquí pudimos atender a lo hecho -decisión política y participación ciudadana-, lo dicho y lo escrito -ciertas metáforas políticas- por el proyecto político kirchnerista en 
el proceso de aprobación de la LIG. Así, reparación-inclusión-igualdad conforman un conjunto de metáforas políticas que permiten construir legitimaciones ex post ${ }^{18}$ sobre la política analizada. Hablamos de legitimaciones ex post, en primer lugar, porque en el caso de la LIG la demanda surgió desde sectores de la ciudadanía organizados en diversas agrupaciones junto a otros sectores políticos (el Partido Socialista, especialmente), y fue luego inscripto dentro del proyecto político kirchnerista. De igual manera, entendemos que no sería posible pensar que los derechos de diversidad sexual se encontraban en el programa político del kirchnerismo -suponiendo que tal programa hubiera existido. Sin embargo, el grado de abstracción propio de las metáforas permite que sean incorporadas políticas muy diversas dentro de este proyecto político.

Esta práctica logró ser legitimada a partir del mapa de ideas que esbozamos anteriormente. En primer lugar, remarcando el respeto de los lineamientos liberales propios de un Estado de Derecho, que es posible rastrear en los discursos de los sectores organizados que analizamos. Junto a la presencia de planteos liberalkantianos, se evidencia la evocación nacional-popular que tiene en el Estado su actor principal. Un Estado que acciona para reparar los daños sociales que ha sufrido el colectivo trans, y cuyas metáforas de la inclusión e igualdad permitieron la resignificación de la demanda de la sociedad civil.

Nos referimos a resignificación ya que el mantenimiento de los argumentos ciudadanos se dio siempre que fue posible incorporarlos dentro de las metáforas que nombramos. En este sentido, el rol del Estado en relación con los derechos humanos es una muestra del mantenimiento del planteo liberal que se compatibilizó con una figura estatal activa. Para ello, fue fundamental la intermediación de la inclusión y la igualdad entre planteos provenientes de diferentes tradiciones políticas. Estos elementos nos permiten pensar que la centralidad de la figura estatal dentro de la tradición nacionalpopular es la que habilita procesos de resignificación de elementos propios de otras corrientes políticas, así como variadas demandas provenientes desde la sociedad civil. En el proceso puntual de la Ley de Identidad de Género, entendemos que no hubiera sido posible mantener elementos propios de la concepción liberal-kantiana sobre el Estado, por lo que operaron dichos procesos para hacer compatible el aseguramiento de la autodeterminación individual con una figura estatal no abstencionista. Las metáforas políticas que enumeramos permitieron esta mixtura.

\section{Bibliografía}

Beriso, D., \& Quintana, M. M. (2010). Derechos humanos: fundamentos filosóficos y perspectivas políticas. En B. Pereyra, \& P. Vommaro, Movimientos sociales $y$ derechos humanos en Argentina (págs. 79-106). Buenos Aires: CICCUS.

\footnotetext{
${ }^{18}$ Este planteo pertenece a Muñoz y Retamozo (2012).
} 
Bidart Campos, G. (2006). Manual de la Constitución Reformada (Vol. I). Buenos Aires: Ediar.

Bobbio, N. (1995). Igualdad y libertad. Madrid: Paidós.

Cheresky, I. (1998). La ciudadanía, la opinión pública y los medios de comunicación. Ciudadanía y política en la Argentina de los 90. Biblioteca Virtual Clacso, disponible en http://biblioteca.clacso.edu.ar/ar/libros/lasa98/Cheresky.pdf.

Corrales, J., \& Pecheny, M. (30 de julio de 2010). Six Reasons Why Argentina Legalized Gay Marriage First. Recuperado el 10/10/2014, de Americas Quarterly: http://americasquarterly.org/node/1753

Franzé, J. (2014). La política, ¿administración o creación? En J. Franzé, Democracia: ¿consenso o conflicto? Agonismo y teoría deliberativa en la política contemporánea. Madrid: Catarata.

Gutiérrez, F. (2011). Poder y democracia en Claude Lefort. Revista de Ciencia Política, 31(2), 247-266.

Hassner, P. (2009). Immanuel Kant. En L. Strauss, \& J. Cropsey, Historia de la filosofía política (págs. 549-584). México: Fondo de Cultura Económica.

Kant, I. (2008). Teoría y praxis. Buenos Aires: Prometeo Libros.

Leclerc, V. (2014). Sistematización del proceso para la aprobación de la Ley de Identidad de Género en Argentina. Programa de Naciones Unidas para el Desarrollo.

Lefort, C. (2011). Democracia y representación. En C. Lefort, Democracia y representación (págs. 19-32). Buenos Aires: Prometeo Libros.

Marchart, O. (2009). El pensamiento político posfundacional. La diferencia política en Nancy, Lefort, Badiou y Laclau. Buenos Aires: Fondo de Cultura Económica.

Muñoz, M. A., \& Retamozo, M. (5 al 7 de diciembre de 2012). Kirchnerismo: gobierno, política y hegemonía. Recuperado el 15/10/2014, de VII Jornadas de Sociología de UNLP: http://www.memoria.fahce.unlp.edu.ar/trab_eventos/ev.2215/ev.2215.pdf

Retamozo, M. (2009). Sujetos políticos: teoría y epistemología. Un dialogo entre la teoría del discurso, el (re)constructivismo y la filosofía de la liberación. XXVII Congreso de la Asociación Latinoamericana de Sociología. Buenos Aires: Asociación Latinoamericana de Sociología. Recuperado el 03/02/2015, de http://www.aacademica.com/000-062/1186

Rinesi, E. (2011a). ¿Qué es el kirchnerismo? En R. (. Hamawi, Qué es el kirchnerismo: escritos desde una época de cambio (págs. 27-40). Buenos Aires: Continente.

Rinesi, E. (2011b). Notas para una caracterización del kirchnerismo. Debates y Combates(1), 141-171.

Salas Oroño, A. (2011). El kirchnerismo como proyecto político y como sociabilización. En R. S. Hamawi, Qué es el kirchnerismo. Escritos desde una época de cambio. Buenos Aires: Continente. 


\section{Fuentes periodísiticas}

Diario Página 12. (2009/10/13). Curarse en salud. Recuperado el 2014/09/15, de http://www.pagina12.com.ar/diario/suplementos/soy/1-1047-2009-10-24.html

Diario Página 12. (2010/09/03). El lanzamiento de la mesa redonda. Recuperado el 2014/09/16, de www.pagina12.com.ar/diario/sociedad/3-152490-2010-0903.html

Diario Página 12. (2011/08/07). Identidad de Género. Recuperado el 16/10/2014, de http://www.pagina12.com.ar/diario/sociedad/3-174676-2011-08-17.html

Diario Página 12. (2011/11/06). En un reclamo de identidad. Recuperado el 18/10/2014, de http://www.pagina12.com.ar/diario/sociedad/3-180693-2011-11-07.html

SentidoG. (2010/08/18). Comenzó el debate por la Ley de Identidad de Género en Diputados. Recuperado el 20/10/2014, de http://www.sentidog.com/lat/2011/08/comenzo-el-debate-por-la-ley-deidentidad-de-genero-en-diputados.html

\section{Documentos Oficiales}

Argentina. Cámara de Diputados de la Nación (16/11/2007). Proyecto: Ley de Identidad de Género. Exp. 5259-D-2007. Recuperado el 20 de octubre de 2014, de http://www1.hcdn.gov.ar/proyxml/expediente.asp?fundamentos $=s i \&$ numexp $=52$ 59-D-2007

Argentina. Cámara de Diputados de la Nación (01/10/2010). Proyecto: Ley de Identidad de Género. Exp. 7243-D-2010. Recuperado el 20 de octubre de 2014, de http://www1.hcdn.gov.ar/proyxml/expediente.asp?fundamentos $=$ si\&numexp $=72$ 43-D-2010.

Argentina. Cámara de Diputados de la Nación (19/10/2010). Proyecto: Ley de reconocimiento y respeto a la identidad de Género. Exp. 7644-D-2010. Recuperado el 20 de octubre de 2014, de http://www1.hcdn.gov.ar/proyxml/expediente.asp?fundamentos=si\&numexp $=76$ 44-D-2010.

Argentina. Cámara de Diputados de la Nación (10/11/2010). Proyecto: Régimen para el reconocimiento y respeto a la identidad de Género. Exp. 8126-D-2010. Recuperado el 20 de octubre de 2014, de http://www1.hcdn.gov.ar/proyxml/expediente.asp?fundamentos $=$ si\&numexp $=81$ 26-D-2010.

Argentina. Cámara de Diputados de la Nación (30/11/2011). Versión Taquigráfica. 10 Reunión - $7^{\circ}$ Sesión Ordinaria (Especial). Período 129. Recuperado el 24 de octubre de 2014, de http://www1.hcdn.gov.ar/sesionesxml/reunion.asp?p=129\&r=10.

Argentina. Cámara de Senadores de la Nación (09/05/2012). Versión Taquigráfica. $5^{\circ}$ Reunión - $3^{\circ}$ Sesión ordinaria. Período $130^{\circ}$. Recuperado el 25 de octubre de 
2014, de http://www.senado.gov.ar/parlamentario/sesiones/2012-059\%2000\%3A00\%3A00/05/downloadTac.

Fernández, C. (2012/07/02). Acto de entrega de DNI con reconocimiento del derecho a la identidad: Palabras de la Presidenta de la Nación. Recuperado el 20/11/2014, de Página Oficial de la Presidencia de la Nación: http://www.presidencia.gob.ar/discursos/25958-acto-de-entrega-de-dni-conreconocimiento-del-derecho-a-la-identidad-palabras-de-la-presidenta-de-lanacion. 